\title{
Useful defects in silicon carbide
}

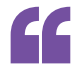

We think that

this interface should allow

us to prepare the spins into highly pure

quantum

states, create entanglement between the spins and light, and detect the spins with high fidelity

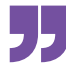

A high-fidelity interface between spins and photons - an essential component of quantum networks, which are in turn needed to enable quantum computers - can be realized using defects in silicon carbide films, David Awschalom and colleagues report in Physical Review X. Defects in semiconductors can exhibit electronic spin states with long coherence times - that is, the spins can retain their orientation for a sufficiently long time. The spin states can be initialized and detected optically, enabling their use for the storage and manipulation of information; thus, these defects hold promise as qubits, the quantum analogues of classical bits. Qubits can be connected to form a network that allows the transmission of information between them: a quantum network. Such a network requires the conversion of quantum information from the spin of the defect into light (that is, to correlate the quantum states of the spin and photons or, in more technical language, to entangle them).

Nitrogen-vacancy (NV) centres in diamond are the most studied type of defect in semiconductors. They are attractive as candidate qubits because they can be manipulated individually, have long spin coherence times and exhibit a set of optical transitions that can serve as a good interface between the defect's spin

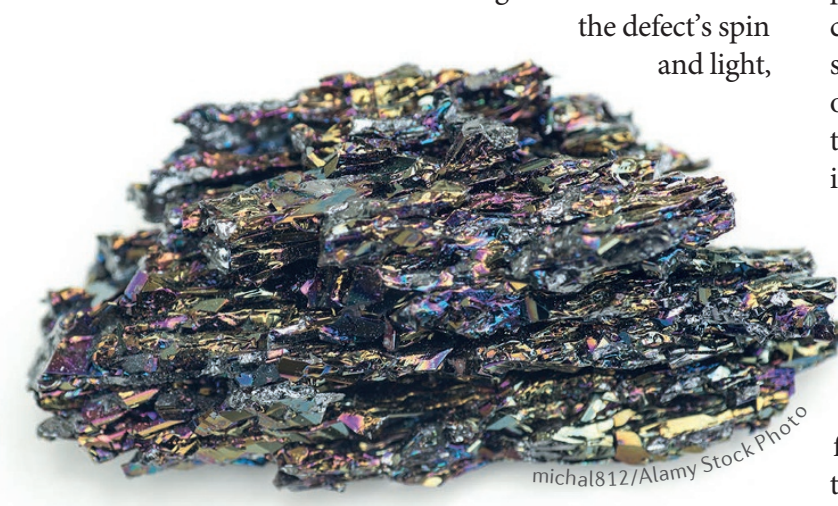

enabling the initialization and readout of the qubits and the transmission of information. However, NV centres emit in the visible range, which results in high losses in the fibres that connect the qubits. "A few years ago, our group started wondering if the NV centre was unique, and whether other defects in different materials might behave in a similar way," says Awschalom. "It would be ideal if these other defects were in a more flexible material platform than diamond, which is hard to grow and microfabricate, and if they were superior to the NV centre for certain applications." Over the past years, the researchers used density functional theory modelling of defects in solid state systems to identify new materials in which it would be possible to create and control quantum states. Divacancy defects in silicon carbide - consisting of a missing Si atom next to a missing $\mathrm{C}$ atom - emerged as a promising candidate. The features that make defects in silicon carbide particularly interesting as potential qubits include the host material's commercial availability, the ease of microfabrication and integration into devices (a particular form of silicon carbide can even be epitaxially grown on silicon), and their emission in the telecom range. Because their optical properties are similar to those of NV centres, protocols developed for that system will be applicable. Material optimization is essential in controlling these quantum states, because low impurity and low strains are needed.

The optical transitions of

divacancies are extremely spin selective and allow detection of the spin state with $94 \%$ visibility; the researchers envisage that improving this metric even further will be possible. "Based on this high spin selectivity, we think that this interface should allow us to prepare the spins into highly pure quantum states, create entanglement between the spins and light, and detect the spins with high fidelity," explains David Christle, first author of the study. "If these defects are to function as elements in a quantum network, each of these three things is a requirement."

The first two requirements were met in previous work by the group. "Now, we filled in the missing link of a good interface between the spin and light; this interface functions near the telecom wavelengths, which means it should have a tenfold reduction of losses in fibre compared with diamond NVs," continues Christle. "We also found that although the interface functions in a similar way to that of NV centres, it actually works better. Based on our measurements, we predict that we could measure the quantum state with an error rate that is ten times lower than that for NV centres."

The next step will be to improve the efficiency in collecting the photons that come out of the defects - more photons mean fewer errors. After that, the goal will be to entangle divacancies separated by macroscopic distances (a few metres) over an optical fibre. "We should eventually be able to entangle spin states over longer distances than what has been done in diamond $(\sim 1.3 \mathrm{~km})$ because of the lower losses inside the fibre when working with near-telecomwavelength light. The tenfold reduction in losses means we could entangle at ten times the distance, all other things being equal," concludes Awschalom.

Giulia Pacchioni

ORIGINAL ARTICLE Christle, D. J. et al. Isolated spin qubits in $\mathrm{SiC}$ with a high-fidelity infrared spinto-photon interface. Phys. Rev. X 7, 021046 (2017) 